\title{
Added value gated PET with phase analysis for the detection of scar burden and prognostication in cardiac sarcoidosis?
}

\author{
Thomas H. Schindler, MD, PhD, ${ }^{\mathrm{a}, \mathrm{b}}$ Adeel Haq, $M D,{ }^{\mathrm{a}}$ and Sudhir Jain, $\mathrm{MD}^{\mathrm{b}}$ \\ a Division of Nuclear Medicine, Mallinckrodt Institute of Radiology, Washington University \\ School of Medicine, St. Louis, MO \\ b Cardiovascular Division, John T. Milliken Department of Internal Medicine, Washington \\ University School of Medicine, St. Louis, Missouri
}

Received Jan 2, 2021; accepted Jan 4, 2021

doi: $10.1007 / \mathrm{s} 12350-021-02530-\mathrm{z}$

\section{See related article, pp. 1389-1401}

Sarcoidosis is known as a systemic autoimmune disease with non-caseating granuloma that can manifest in any organs with preference for perihilar-mediastinal lymph nodes and the lungs. ${ }^{1,2}$ Interestingly, a substantial variability in the prevalence of sarcoidosis ranging between 3 and 10 per 100,000 for Caucasians (highest in Scandinavians), from 35 to 80 per 100,000 African American has been noted. ${ }^{1,2}$ Further, there is an increasing awareness that the life span of patients with sarcoidosis is increasingly stringent on the cardiac involvement. Notably, the prevalence of cardiac involvement in sarcoidosis in autopsy studies has been appreciated between 20\% and 27\% in the United States population and even up to $58 \%$ in Japan. ${ }^{1,3}$ While cardiac sarcoidosis may not become apparent as a subclinical bystander to systemic disease activity, it can induce acute-inflammatory myocardial disease activity that potentially may result into mild fibrotic alterations and/or even scar tissue. ${ }^{3}$ Clinically, cardiac sarcoidosis may manifest with complete heart block, ventricular arrhythmias (tachycardia and/or fibrillation), sudden cardiac death, and congestive heart failure. ${ }^{4,5}$ Given the potential adverse outcome of clinically manifest cardiac

Reprint requests: Thomas H. Schindler, MD, PhD, Division of Nuclear Medicine, Mallinckrodt Institute of Radiology, Washington University School of Medicine, 510 S. Kingshighway, St. Louis, MO 63110; thschindler@wustl.edu

J Nucl Cardiol 2022;29:1402-4.

$1071-3581 / \$ 34.00$

Copyright (c) 2021 American Society of Nuclear Cardiology. sarcoidosis, its early and accurate detection is of utmost importance. With the advent of advanced imaging modalities such as cardiac positron emission tomography (PET), magnetic resonance (MR) imaging or as integrated PET/MR, ${ }^{6-9}$ cardiac sarcoidosis can be detected and characterized non-invasively that implies critical prognostication and a timely installation of immune-suppressive treatment options. ${ }^{2}$ As regards perfusion and FDG-PET imaging, the typical location of resting perfusion deficits (basal segments; commonly affecting the septum or lateral wall of the left ventricle) with corresponding abnormal focal FDG-uptake, so called "mismatch", is widely diagnostic for active-inflammatory cardiac sarcoidosis., ${ }^{2,10}$ Importantly, such focal regions of "mismatch" between perfusion and inflammation may be related to regional leukocyte infiltration with associated inflammation, edema, and/or fibrosis. Surprisingly, such regions may have maintained or normal left ventricular wall motion that contrast classical regional hypo-to-akinesis in infarcted tissue after acute coronary syndromes. ${ }^{2,11}$ The reason for this discordant observations remain uncertain but may be related to relatively low spatial resolution of SPECT or PET perfusion imaging, edema induced compression of the coronary arteriolar vessels, low grade of interstitial fibrosis and/or patchy pattern of scar tissue (if present). In addition, compensatory increases in regional contractility within the affected wall segments may also account for the observed normal transmural wall motion on gated PET perfusion images. ${ }^{12}$ In this respect, adding phase analysis on gated single photon emission computed tomography, assessing the heterogeneity of perfusion deficits, and the dyssynchrony of the left ventricular wall motion in cardiac sarcoidosis, may be of further help. ${ }^{11,13}$ 
In this issue of the Journal of Nuclear Cardiology, Elwazir et al. ${ }^{14}$ could indeed demonstrate that adding phase analysis to gated PET-derived volume and wall motion resulted in an improved detection of sarcoidosisrelated regional scar tissue superior to perfusion assessment with ${ }^{13} \mathrm{~N}$-ammonia images alones. As it was observed, the sensitivity and specificity of ${ }^{13} \mathrm{~N}$-ammonia perfusion images was about $45 \%$ and $89 \%$, respectively. Adding, PET-derived left ventricular volumes and function in a logistic regression model enhanced the sensitivity to $57 \%$ with a drop in specificity to $80 \%$. Importantly, subsequent evaluation with phase analysis with maximal segmental onset of myocardial contraction $>61$ resulted in an improvement of the AUC from .72 to .75 . This resulted in a relabeling of $16.3 \%$ of patients as scar with a net reclassification index of $8.2 \%$.

Overall, 176 patients underwent delayed enhancement (DE) cardiac magnetic resonance (CMR) and also ${ }^{13} \mathrm{~N}$-ammonia rest perfusion combined with ${ }^{18} \mathrm{~F}$-fluorodeoxyglucose (FDG) cardiac PET/CT for evaluation of patients with suspicion for cardiac sarcoidosis. Scar was defined as DE on CMR without concordant ${ }^{18} \mathrm{~F}$ FDG uptake on 18F-FDG uptake. The latter definition to define scar tissue by DE-CMR without corresponding ${ }^{18}$ F-FDG uptake may be suboptimal as DE on CMR images could also represent, at least in part, edema in particular in the absence of regional wall motion abnormality. The additional application of non-contrast T2-weighted CMR sequences would certainly have been helpful to reliably identify myocardial edema in these patients with cardiac sarcoidosis. Conversely, there is an ongoing debate whether T2-weighted CMR sequences may accurately differentiate between myocardial edema and scar tissue with DE on T1-weighted CMR images when of preexisting sarcoidosis-induced cardiac structural alterations are present. ${ }^{7,15}$

Interestingly, in the group with apparent scar tissue the global left ventricular ejection fraction (LVEF), as determined with cine CMR, was in the normal range with a mean value of $56 \%$ and a standard deviation of $14 \%$. It would have been of interest to subgroup the group with "defined" scar tissue into those with regional wall motion abnormality and in those without. It is quite possible that fibrotic changes or minor scar tissue islets were compensated by increase in regional circumferential contractility so that on gated PET it appeared as normal regional transmural wall motion. In this respect, it may be of interest to apply tagging CMR for the assessment circumferential, radial, and/or longitudinal strain in order to evaluate the accuracy of this approach in the detection of regional contractile abnormalities when gated PET appears to be normal in regions with DE on CMR images. Someone could argue that tagging CMR could indeed be helpful to differentiate between edema and minor scar burden in these regions with cardiac DE that, however, needs to be investigated. In the current study, Elwazir et al. ${ }^{14}$ elegantly demonstrate that adding phase analysis to gated PET-derived volume and wall motion may indeed increase the detection of regional scar tissue with an AUC of about .75 that led to a relabeling of $16 \%$ of patients with sarcoidosis related scar burden. Given that the residual scar burden after active-inflammatory cardiac sarcoidosis is a major and independent long-term predictor in clinical outcome in these patients, ${ }^{5}$ the additional application of phase analysis with gated ${ }^{13} \mathrm{~N}$ ammonia PET may hold promise for a further improvement in prognostication of cardiac sarcoidosis patients that deserves further investigations in largescale clinical trials.

\section{Disclosure}

This work is supported by a departmental fund from Washington University (No. 12-3271-93128). Dr. Schindler has received research support from NIH as MPI for 1RO1HL142297-01A1 and from GE Healthcare (GE-265303).

\section{References}

1. Chareonthaitawee P, Beanlands RS, Chen W, Dorbala S, Miller EJ, Murthy VL, et al. Joint SNMMI-ASNC expert consensus document on the role of (18)F-FDG PET/CT in cardiac sarcoid detection and therapy monitoring. J Nucl Cardiol. 2017;24:174158.

2. Kruse MJ, Kovell L, Kasper EK, Pomper MG, Moller DR, Solnes $\mathrm{L}$, et al. Myocardial blood flow and inflammatory cardiac sarcoidosis. JACC Cardiovasc Imaging. 2017;10:157-67.

3. Kumita S, Yoshinaga K, Miyagawa M, Momose M, Kiso K, Kasai $\mathrm{T}$, et al. Committee for diagnosis of cardiac sarcoidosis using 18FFdg Pet JSoNC Recommendations for (18)F-fluorodeoxyglucose positron emission tomography imaging for diagnosis of cardiac sarcoidosis-2018 update: Japanese Society of Nuclear Cardiology recommendations. J Nucl Cardiol. 2019;26:1414-33.

4. Schindler TH, Valenta I. Isolated cardiac sarcoidosis-a rare disease entity? Int J Cardiol. 2018;253:194-5.

5. Schindler TH, Derenoncourt P, Leucker TM. Cardiac sarcoidosis and prediction of sudden death: An ongoing clinical dilemma? Int J Cardiol 2020. https://doi.org/10.1016/j.ijcard.2020.12.035.

6. Schindler TH, Bateman TM, Berman DS, Chareonthaitawee P, De Blanche LE, Dilsizian V, et al. Appropriate use criteria for PET myocardial perfusion imaging. J Nucl Med. 2020;61:1221-65.

7. Schindler TH. Cardiovascular PET/MR imaging: Quo Vadis? J Nucl Cardiol. 2017;24:1007-18.

8. Garcia EV, Slomka PJ, Moody JB, Germano G, Ficaro EP. Quantitative clinical nuclear cardiology. Part 1: Established applications. J Nucl Cardiol. 2020;27(1):189-201. https://doi.org/ 10.1007/s12350-019-01906-6.

9. Slomka PJ, Moody JB, Miller RJH, Renaud JM, Ficaro EP, Garcia EV. Quantitative clinical nuclear cardiology, part 2: Evolving/ emerging applications. J Nucl Cardiol. 2020. https://doi.org/10. 1007/s12350-020-02337-4. 
10. Benetos G, Giannopoulos AA. Myocardial perfusion imaging in cardiac sarcoidosis: A "sine qua non" for prognosis assessment? J Nucl Cardiol. 2019. https://doi.org/10.1007/s12350-019-01964-w.

11. Koyanagawa K, Naya M, Aikawa T, Manabe O, Furuya S, Kuzume M, Oyama-Manabe N, Ohira H, Tsujino I, Anzai T. The rate of myocardial perfusion recovery after steroid therapy and its implication for cardiac events in cardiac sarcoidosis and primarily preserved left ventricular ejection fraction. J Nucl Cardiol. 2019. h ttps://doi.org/10.1007/s12350-019-01916-4.

12. Vincenti GM, Ambrosio G, Hyacinthe JN, Quercioli A, Seimbille $\mathrm{Y}$, Mach F, et al. Matching between regional coronary vasodilator capacity and corresponding circumferential strain in individuals with normal and increasing body weight. J Nucl Cardiol. 2012;19:693-703.

13. Koyanagawa K, Naya M, Aikawa T, Manabe O, Kuzume M, Ohira H, Tsujino I, Tamaki N, Anzai T. Prognostic value of phase analysis on gated single photon emission computed tomography in patients with cardiac sarcoidosis. J Nucl Cardiol. 2019. https://doi. org/10.1007/s12350-019-01660-9.

14. Elwazir M, Bird J, AbouEzzeddine O, Chareonthaitawee P, Blauwet L, Collins J, Gibbons R, et al. Performance of cardiac PET/CT with and without phase analysis for detection of scar in cardiac sarcoidosis: Comparison to cardiac magnetic resonance imaging. J Nucl Cardiol 2021 (in press).

15. Kim HW, Van Assche L, Jennings RB, Wince WB, Jensen CJ, Rehwald WG, et al. Relationship of T2-Weighted MRI myocardial hyperintensity and the ischemic area-at-risk. Circ Res. 2015;117:254-65.

Publisher's Note Springer Nature remains neutral with regard to jurisdictional claims in published maps and institutional affiliations. 